Radiologe $2018 \cdot 58: 278-280$

https://doi.org/10.1007/s00117-018-0381-9

(c) Springer Medizin Verlag GmbH, ein Teil von Springer Nature 2018

CrossMark

M. Scharitzer · T. Mang · C. Herold

Universitätsklinik für Radiologie und Nuklearmedizin, Medizinische Universität Wien, Wien, Österreich

\title{
Radiologische Bildgebung der entzündlichen Erkrankungen des Gastrointestinaltrakts
}

Liebe Leserinnen und Leser,

in der vorliegenden Ausgabe von Der Radiologe wird die radiologische Bildgebung der entzündlichen Erkrankungen des Gastrointestinaltrakts und ihrer Komplikationen schwerpunktmäßig behandelt.

In den letzten Jahren ist es bei der radiologischen Diagnostik gastrointestinaler Erkrankungen zu substanziellen Verbesserungen mit einer Erweiterung des Indikationsspektrums gekommen. Insbesondere bei chronisch-entzündlichen Erkrankungen stieg durch die zunehmende Entwicklung spezifischerer Therapieverfahren der Bedarf an radiologischer Diagnostik und bildbasierter Kontrolle des Behandlungserfolgs. Dabei konnte ein Wandel der verwendeten radiologischen Modalitäten beobachtet werden. Obwohl konventionelle Bildgebungsverfahren in manchen Bereichen, wie beispielsweise dem Schluckröntgen, nach wie vor einen wesentlichen Stellenwert einnehmen, hat ihre Bedeutung insgesamt abgenommen. Doppelkontrastuntersuchungen wie das Magenröntgen, das Enteroklysma nach Herlinger und Sellink sowie der Kolonkontrasteinlauf werden in vielen radiologischen Abteilungen selten oder gar nicht mehr durchgeführt. Dadurch ist es für Ärzte in radiologischer Ausbildung zunehmend schwieriger, die notwendigen Fertigkeiten $\mathrm{zu}$ erlernen, um eine Doppelkontrastuntersuchung bei entsprechender Indikation mit ausreichender Expertise und Qualität durchführen zu können. Gleichzeitig hat besonders die moderne Schnittbildgebung mittels Computertomographie (CT) und Magnetresonanz- tomographie (MRT) die Bildgebung entzündlicher Veränderungen des Gastrointestinaltrakts revolutioniert. Dies zeigt sich vor allem bei chronischentzündlichen Darmerkrankungen in beginnendem Umdenken hinsichtlich des therapeutischen Ansatzes. Im Gegensatz zur ausschließlichen Bewertung der Mukosa wird nun auch eine nur mittels Schnittbildverfahren beurteilbare transmurale Heilung entzündlicher Darmveränderungen als Behandlungsziel diskutiert.

Dieses Themenheft soll unterschiedliche bildmorphologische Kriterien aufzeigen, welche die Diagnose, Charakterisierung und Graduierung entzündlicher Wandveränderungen des Gastrointestinaltrakts ermöglichen. Der Schwerpunkt liegt dabei auf der Wahl der richtigen Untersuchungsmethode, ihrer korrekten Anwendung, der Interpretation und Klassifikation gefundener Pathologien sowie auf der Kenntnis von Differenzialdiagnosen und Fehlermöglichkeiten bei der Befundung.

Die Beiträge umfassen eine systematische Analyse verschiedener Muster und Zeichen entzündlicher Wandveränderungen des Gastrointestinaltrakts, die radiologische Diagnostik akuter und chronisch-entzündlicher Erkrankungen des oberen sowie des unteren Gastrointestinaltrakts und die Anforderungen an die Radiologie aus chirurgischer und internistischer Sicht. Die Diagnostik entzündlicher gastrointestinaler Pathologien des pädiatrischen Patientengutes wird ebenso separat behandelt wie die Abklärung und Klassifikation der Divertikelerkrankung und perianal entzündlicher Veränderungen. 
Die Herausgeber und Autoren hoffen, dem Leser mit dieser Ausgabe, die sowohl den Charakter eines praxisorientierten Leitfadens als auch den einer Zusammenfassung neuester Entwicklungen aufweist, aktuelle Informationen zu den verschiedenen Methoden der modernen bildgebenden Diagnostik gastrointestinaler Entzündungen in die Hand zu geben.

Ihre

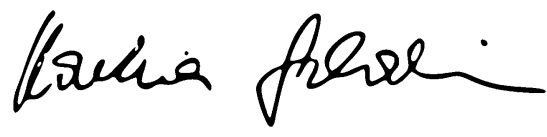

Ass.-Prof. Dr. Martina Scharitzer

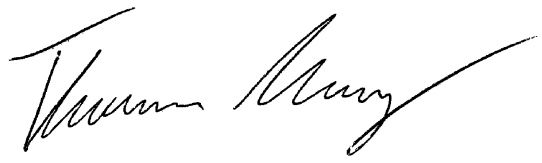

Assoc.-Prof. PD Dr. Thomas Mang

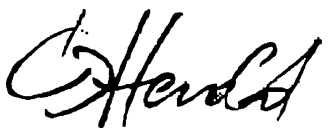

Prof. Dr. Christian Herold

\section{Korrespondenzadresse}

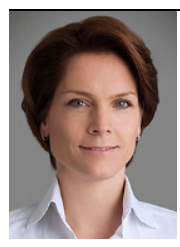

Ass.-Prof. Dr. M. Scharitzer

Universitätsklinik für Radio-

logie und Nuklearmedizin,

Medizinische Universität

Wien

Währinger Gürtel 18-20,

1090 Wien, Österreich

martina.scharitzer@

meduniwien.ac.at

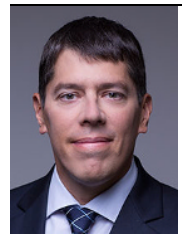

Assoc.-Prof. PD Dr. T. Mang

Universitätsklinik für Radio-

logie und Nuklearmedizin,

Medizinische Universität

Wien

Währinger Gürtel 18-20,

1090 Wien, Österreich

thomas.mang@

meduniwien.ac.at

Interessenkonflikt. M. Scharitzer, T. Mang und C. Herold geben an, dass kein Interessenkonflikt besteht.

\section{Früher gegensteuern bei unwirksamen Therapien}

Im Verbundprojekt „PANTHER“ arbeiten Wissenschaftler daran, CT-Bilder effektiver als heute für die Verlaufskontrolle von Tumorbehandlungen einzusetzen.

Heute überprüfen Mediziner den Verlauf einer Tumortherapie, indem sie den Allgemeinzustand ihrer Patienten beobachten, Laborwerte aus Blutuntersuchungen analysieren und in regelmäßigen Abständen CT-Aufnahmen der betroffenen Organe machen. Bislang orientieren sich die Ärzte auf diesen Bildern an der Größenentwicklung des Tumors: Ist er in Folge einer Strahlenbehandlung oder einer Chemotherapie geschrumpft? Oder wächst er womöglich weiter, sodass ein Therapiewechsel sinnvoll erscheint, zum Beispiel die Wahl eines anderen Medikaments?

Hier setzt das Verbundprojekt PANTHER an. Bislang ist die Größenentwicklung des Tumors das wichtigste Kriterium bei der Beurteilung der CT-Bilder, doch in den Aufnahmen stecken noch viel mehr Informationen, die aber bislang kaum genutzt werden. So zeigen die Bilder zusätzlich zum Größenverlauf, ob und wie sich die Form eines Geschwürs im Laufe der Zeit verändert. Außerdem können sie Details über die Beschaffenheit des Tumors liefern: Besteht er aus verschiedenen Gewebearten, und verändert sich seine Zusammensetzung im Laufe der Therapie?

Mit bloßem Auge sind viele dieser Zusatzinformationen nicht zu sehen. Um diese Merkmale erkennen und vor allem quantifizieren zu können, wird eine Computerunterstützung benötigt und genau das ist das Ziel von PANTHER. Das Verbundprojekt „Patientenorientierte onkologische Therapieunterstützung" wird seit Oktober 2016 vom Bundesministerium für Bildung und Forschung gefördert und umfasst ein Projektvolumen von knapp 2,8 Millionen Euro. Neben Fraunhofer MEVIS sind das Klinikum der Universität München, MeVis BreastCare $\mathrm{GmbH} \&$ Co.KG sowie als Projektkoordinator Siemens Healthcare $\mathrm{GmbH}$ beteiligt.

In einem ersten Schritt stellten Radiologen der Uniklinik München große Mengen an CTBilddaten von Darmkrebs- und LymphomPatienten zusammen. In diesen Daten haben die Kliniker die relevanten Strukturen mit Hilfe eines Webtools segmentiert, also eingegrenzt. Dadurch sind Größe und Form der Tumoren bzw. Organe in den Aufnahmen klar zu erkennen und quantitativ zu vermessen. Im späteren Projektverlauf soll diese Segmentierung automatisch durch einen Algorithmus erfolgen.

Ferner stellten Onkologen aus der Münchener Klinik umfangreiches Datenmaterial darüber zur Verfügung, wie die Patienten auf eine Therapie angesprochen haben und wie sich ihre Blutwerte im Laufe der Behandlung entwickelten. Diese Daten wollen die Experten nun mit bestimmten Merkmalen in den CT-Bildern abgleichen, etwa wie sich Form und Textur eines Tumors im Laufe einer Therapie verändern. Umfangreiche statistische Analysen sollen verraten, ob und an welchen Stellen es verlässliche Zusammenhänge zwischen Bild- und Therapiedaten gibt. Im Idealfall läst sich anhand der CT-Bilder früher als bislang abschätzen, ob die eingeschlagene Therapie Erfolg hat oder nicht.

Zum Projektende im Herbst 2019 sollte klar sein, welchen Nutzen dieser Ansatz für die Medizin bringen kann. Der nächste Schritt wäre dann die Entwicklung eines computerbasierten Expertensystems, das die Ärzte bei der Suche nach der besten Therapie unterstützt.

Quelle: Fraunhofer MEVIS, Bremen www.mevis.fraunhofer.de 
Hier steht eine Anzeige.

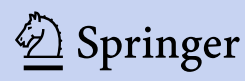

